\title{
2.||||||||||||||||||||||||||||||||||||||||||||||||||||||||||||||||||||.
}

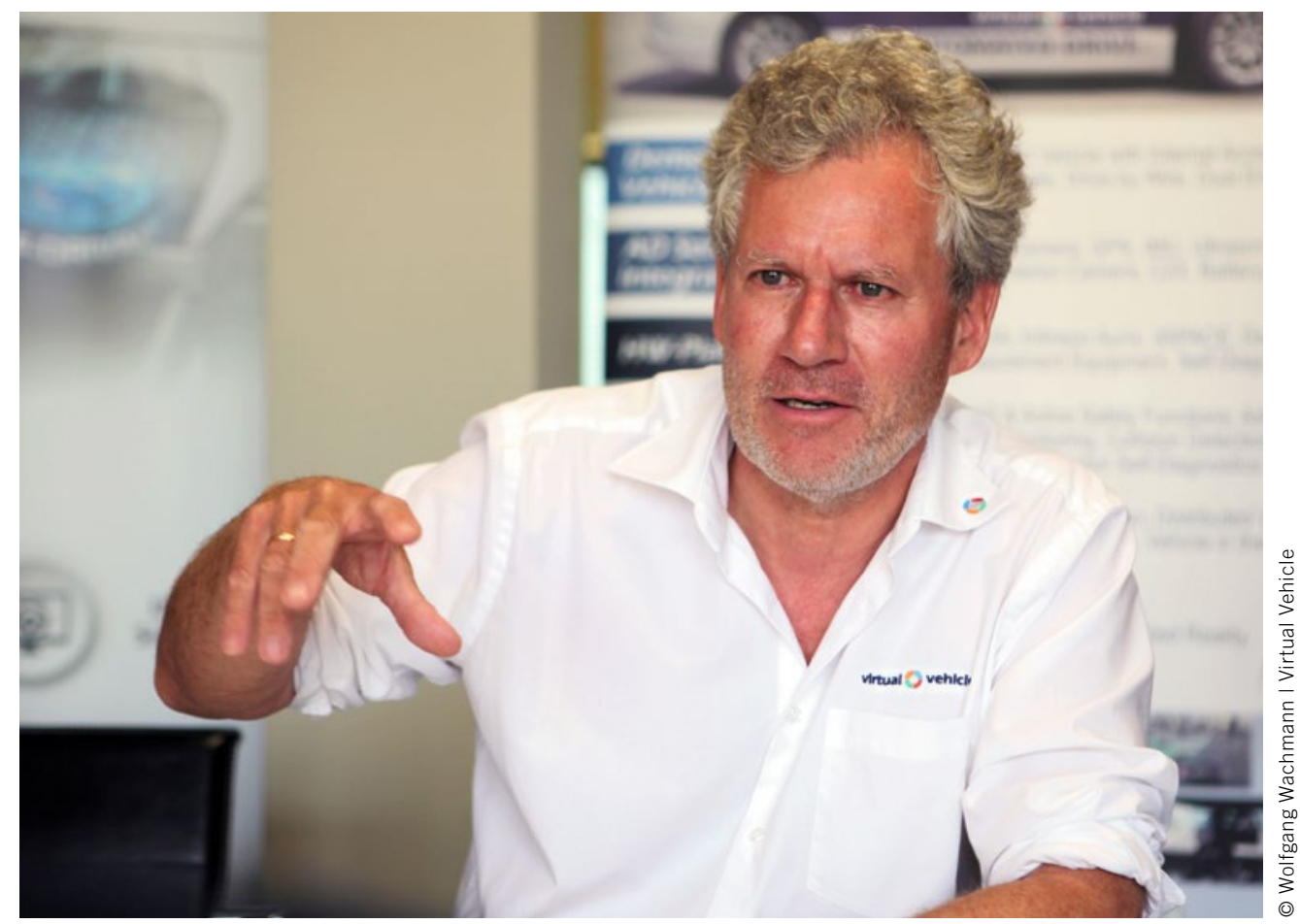

Dr. rer. nat. Jost Bernasch Geschäftsführer der Virtual Vehicle Research GmbH

\section{Die einzige Regel - Software gewinnt}

Im Rennen um die Mobilität von morgen gilt nur eine Regel: Software gewinnt. Ähnlich wie bei Roborace, der internationalen Rennserie für automatisierte Fahrzeuge, wird der Weg zum Sieg mit Nullen und Einsen geschrieben. Die mechanische Fahrzeugstruktur wird zur Selbstverständlichkeit degradiert, die als Verkaufsargument an Schlagkraft verliert. Design und neue Funktionen sind dominant. In Zeiten von Softwaredefined Vehicles werden aus primär mechanischen Objekten global vernetzte, drahtlos (Over-the-Air, OTA) updatefähige Hightech-Fahrsysteme als Teil des Internet of Things - zukünftig Economy of Things genannt.

Tesla ist es gelungen, in diesem Rennen in Führung zu gehen. Doch es ist kein Drag Race, bei dem man mit guter Anfangsbeschleunigung gewinnt. Die Mobilität von morgen ist ein Langstreckenrennen. Alltagstauglichkeit, Komfort und Strategie zählen genauso dazu wie Service, Kundenverständnis und Massenproduktion. Hier punkten klassische OEMs mit ihrer Erfahrung - und mittlerweile sind alle großen Teams in dieses Rennen eingestiegen. Dabei ist klar: Auf der Straße gehen Safety und Security über Speed of Development. Neue E/E-Architekturen mit zentralen Domänenrechnern und neuen Functional-Safetyund Securitykonzepten machen die softwarebasierten Fahrzeuge sicher und alltagstauglich. Zukünftige Softwarearchitekturen sind der Schlüssel für den Wettbewerb der OEMs mit den neuen Fahrzeugherstellern von der grünen Wiese.
Dies alles erfordert einen drastischen Umbau der Entwicklungsprozesse und eine stärker domänenübergreifende Zusammenarbeit. Gesamtsystemdenken gepaart mit kontinuierlicher Erprobung wird die Entwicklung von komplexen Systemen prägen. Die Gesamtsystemsimulation und die funktionsorientierte Entwicklung stehen dabei im Vordergrund und werden durch Ansätze des Model-based Systems Engineering unterstützt. Wie bei Softwareprojekten erfolgreich etabliert, ersetzt die agile Entwicklung eine meilensteingetriebene „Big-Bang Integration“ durch die fortlaufende Integration von Entwicklungsergebnissen und automatisierten Tests. Virtual Vehicle Research unterstützt mehrere Big Player in strategischen Partnerschaften bei dem Paradigmenwechsel für diese neuen Prozesse, Methoden und Technologien. Gemeinsam arbeiten wir an einer neuen Generation von Systemsimulationen mit vertrauenswürdigen, belastbaren und zertifizierbaren Ergebnissen (Credible Simulation). Eine kontinuierliche Integration von Teilsystemen und Daten wird der Schlüssel für Innovations-, Entwicklungs- und Wettbewerbsfähigkeit sein und zukünftige Erfolge sichern.

Das internationale Rennen um die Mobilität der Zukunft hat erst begonnen, doch jeder Meter Vorsprung, der jetzt erreicht wird, ist wesentlich. Denn ein Aspekt dieses Rennens ist klar: Es gibt keine Ziellinie - stattdessen werden zu langsame Teilnehmer gnadenlos überholt und sind aus dem Rennen. 